\title{
Thermodynamics of thallium alkanoates VI. Thallium(I) n-heptanoate revisited ${ }^{a}$
}

\author{
A.K. LABBAN, F. L. LÓPEZ DE LA FUENTE, ${ }^{b}$ J. A. R. CHEDA, \\ EDGAR F. WESTRUM, JR, and F. FERNÁNDEZ-MARTÍN ${ }^{c}$ \\ Department of Chemistry, University of Michigan, \\ Ann Arbor, MI 48109, U.S.A.
}

(Received 2 January 1989)

\begin{abstract}
The heat capacity of thallium(I) $n$-heptanoate has been remeasured on a new sample on which the preparative procedures were modified to eliminate the 1-1 (salt + acid) complex (soap) in a previously studied sample. The sub-ambient heat capacity of a new highly pure thallium(I) $n$-heptanoate is characterized by one set of transitions between 262 and $272 \mathrm{~K}$ and another transition at $301 \mathrm{~K}$. The lowest transition temperature at $262.1 \mathrm{~K}$ has a maximum of $C_{p, m}$ $\approx 559 R$. Next, a bifurcated pair (at 268.6 and $271.4 \mathrm{~K}$ ) have $C_{p, \mathrm{~m}} \mathrm{~s} \approx 300 R$, and the highest transition (at $301.0 \mathrm{~K}$ ) has a maximum $C_{p, \mathrm{~m}} \approx 1697 R$. The corresponding values of $\Delta_{\mathrm{trs}} S_{\mathrm{m}}^{\circ}$ for the two sets are about $2.24 R$ and $1.10 R$. At $298.15 \mathrm{~K}$ the values of $\Delta_{0}^{T} S_{\mathrm{m}}^{\circ}, \Delta_{0}^{T} H_{\mathrm{m}}^{\circ}$, and $\Phi_{\mathrm{m}}^{\circ}(T)$ are $39.79 R, 5979 R \cdot \mathrm{K}$, and $19.74 R$. Smoothed thermodynamic functions at selected temperatures are tabulated through melting.
\end{abstract}

\section{Introduction}

In the course of measurement of the series of thallium alkanoates, ${ }^{(1-4)}$ it was noted subsequent to submission for publication of the results for the thallium(I) $n$-heptanoate ${ }^{(2)}$ that of the five transitions observed in the 260 to $310 \mathrm{~K}$ region, one of the larger ones at $\approx 296 \mathrm{~K}$ was indeed extraneous. Subsequent studies on the phase behavior of the (salt + acid) system revealed ${ }^{(5)}$ that a unimolecular (salt + acid) complex is formed and that several per cent of this contaminated the previous sample. The presence of such 1-1 acid soaps has been demonstrated in alkali laurates, stearates, and oleates by various techniques ${ }^{(6-8)}$ and complexes with higher (salt + acid) ratios have also been claimed.

Subsequent analysis based on $\Delta_{\mathrm{trs}} H_{\mathrm{m}}$ against $x_{\text {salt }}$ values from the corresponding phase diagram, ${ }^{(5)}$ showed the previous sample to contain about $(9 \pm 1)$ moles per cent of acid $^{(2)}$ and to differ from data of Fernández-Martín et al., ${ }^{(9)}$ who did not find the

a The first paper in this series is reference 1 .

${ }^{b}$ Address: Departamento de Química Física, Facultad de Ciencias Quimicas, Universidad Complutense, 28040 Madrid, Spain.

'Address: Instituto del Frío, CSIC, 28040 Madrid, Spain. 
transition at $296.2 \mathrm{~K}$. This paper reports measurements on a new carefully purified sample judged to be 99.7 moles per cent pure and free of the complex. The former III-to-II transition is not present. Consequently, the numbering of phases $>$ III originally designated by Ngeyi et al. ${ }^{(2)}$ must here be reduced by unity. That is, his phase VI becomes V, etc., in the present work. Further studies on the thermophysics of the acid and the 1-1 complex are underway. Previous studies on related systems have been summarized. ${ }^{(10)}$

\section{Experimental}

The sample of T17C used in this study - designated 86-T17C hereafter-was prepared by reacting heptanoic acid (Fluka, puriss grade, $>99$ moles per cent, tested by g.c. at the origin) dissolved in anhydrous methanol with a slight excess $(\approx 5$ per cent) of the stoichiometric amount of $\mathrm{Tl}_{2} \mathrm{CO}_{3}$, suspended in the same solvent. After refluxing under stirring for several hours and separating the excess of $\mathrm{Tl}_{2} \mathrm{CO}_{3}$ by filtration, the clear solution was concentrated until incipient crystallization of the salt occurred and precipitation was completed by adding dry ethyl ether. After filtration, the recovered powdered crystals were purified by repeated recrystallizations from absolute ethanol. The salt was finally vacuum-dried at room temperature to constant mass. I.r. spectroscopic determinations showed no traces of water or free acid. Moreover, several d.s.c. purity determinations by a fractional-fusion technique yielded a mean value of $(99.7 \pm 0.1)$ moles per cent of liquid-soluble solid-insoluble purity. The difference between the method described and that used to synthesize the sample first studied $^{(2)}$ (84-T17C, hereafter) is small. Then, we had followed the method described elsewhere ${ }^{(11)}$ and the advice of Duruz et al. ${ }^{(12)}$ According to them, the decomposition rates for the homologous series of sodium alkanoates are quite high unless a small excess of organic acid is introduced in the reaction medium; they explain that the addition of an excess of acid represses the action of the free hydroxyl ion, considered as the main cause of instability of such salts. Perhaps this assertion is valid only for syntheses in which the water is the solvent - as in the case of Na alkanoates-but even in the case of other compounds prepared in anhydrous methanol, as Cs alkanoates, ${ }^{(13)}$ a slight excess of acid had been also used. Hence, we had also used this method for preparing the $84-\mathrm{T} 17 \mathrm{C}$ sample which became contaminated by heptanoic acid. This, however, could not be demonstrated until careful comparisons were made with other samples of thallium(I) heptanoate, prepared under different conditions, resulting ultimately in a study of the (T17C $+\mathrm{HC} 7)$ phase diagram. ${ }^{(5)}$

\section{CALORIMETRIC PROCEDURE}

Measurements were made from 8 to $350 \mathrm{~K}$ in the adiabatic Mark-X calorimetric cryostat previously described ${ }^{(2,14,15)}$ by an automated intermittent-heating quasiadiabatic technique. ${ }^{(15)}$

The sample loaded into the gold-plated copper calorimeter designated $\mathrm{W}-\mathrm{AB}$ had a mass of $36.419 \mathrm{~g}$ corresponding to a reciprocal amount of substance of $9.1587 \mathrm{~mol}^{-1}$ with molar mass of $333.55 \mathrm{~g} \cdot \mathrm{mol}^{-1}$ (on the basis of the 1978 IUPAC 
atomic masses). The buoyancy corrections were calculated assuming a density of $2.85 \mathrm{~g} \cdot \mathrm{cm}^{-3}$. Following evacuation, $21.0 \mathrm{kPa}$ of purified helium was added to the calorimeter to promote thermal equilibrium.

The thermal history of the thallium(I) $n$-heptanoate is shown by the linear array; solid arrows indicate cooling and dashed arrows indicate results-acquisition (heating) ranges for various series.

$$
\begin{aligned}
& 298 \mathrm{~K} \stackrel{15.7 \mathrm{~h}}{\longrightarrow} 246 \mathrm{~K} \underset{\text { Series I }}{\stackrel{3 \mathrm{~h}}{\rightarrow}} 311 \mathrm{~K} \stackrel{2 \mathrm{~h}}{\longrightarrow} 285 \mathrm{~K} \underset{\text { Series II }}{\stackrel{4.3 \mathrm{~h}}{\rightarrow}} 319 \mathrm{~K} \stackrel{16.3 \mathrm{~h}}{\longrightarrow} 285 \mathrm{~K} \\
& \underset{\text { Series III }}{\stackrel{7 \mathrm{~h}}{\rightarrow}} 320 \mathrm{~K} \stackrel{15.5 \mathrm{~h}}{\longrightarrow} 270 \mathrm{~K} \underset{\text { Series IV }}{\stackrel{4.5 \mathrm{~h}}{\rightarrow}} 314 \mathrm{~K} \stackrel{19 \mathrm{~h}}{\longrightarrow} 270 \mathrm{~K} \underset{\text { Series }}{\stackrel{10.6 \mathrm{~h}}{\rightarrow}} 304 \mathrm{~K}
\end{aligned}
$$

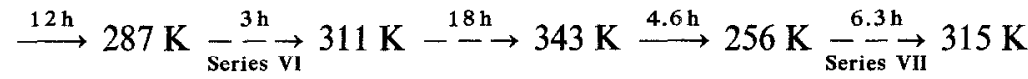

$$
\begin{aligned}
& \stackrel{19.5 \mathrm{~h}}{\longrightarrow} 226 \mathrm{~K} \underset{\text { Series VIII }}{\stackrel{6.3 \mathrm{~h}}{\rightarrow}} 284 \mathrm{~K} \stackrel{23.9 \mathrm{~h}}{\longrightarrow} 232 \mathrm{~K} \underset{\text { Series IX }}{\stackrel{12.7 \mathrm{~h}}{\rightarrow}} 278 \mathrm{~K} \stackrel{2.9 \mathrm{~h}}{\longrightarrow} 230 \mathrm{~K} \\
& \underset{\text { Series } \mathrm{x}}{\stackrel{3 \mathrm{~h}}{\rightarrow}} 282 \mathrm{~K} \stackrel{22.3 \mathrm{~h}}{\longrightarrow} 78 \mathrm{~K} \underset{\text { Series XI }}{\stackrel{8.7 \mathrm{~h}}{\rightarrow}} 160 \mathrm{~K} \stackrel{11 \mathrm{~h}}{\longrightarrow} 153 \mathrm{~K} \underset{\text { Series XII }}{\stackrel{9.1 \mathrm{~h}}{\rightarrow}} 281 \mathrm{~K} \\
& \stackrel{30 \mathrm{~h}}{\longrightarrow} 49 \mathrm{~K} \underset{\text { Series } \mathrm{XIII}}{\stackrel{4.1 \mathrm{~h}}{\rightarrow}} 83 \mathrm{~K} \stackrel{\stackrel{5 \mathrm{~h}}{\longrightarrow}}{\rightarrow} 298 \mathrm{~K} \stackrel{62 \mathrm{~h}}{\longrightarrow} 67 \mathrm{~K} \stackrel{6.3 \mathrm{~h}}{\rightarrow} 38 \mathrm{~K} \\
& \stackrel{2.3 \mathrm{~h}}{\longrightarrow} 7.5 \mathrm{~K} \underset{\text { Series XIV }}{\stackrel{11 \mathrm{~h}}{\rightarrow}} 56.8 \mathrm{~K} \stackrel{13 \mathrm{~h}}{\rightarrow} 307 \mathrm{~K} \underset{\text { Series } \mathrm{xV}}{\stackrel{1.6 \mathrm{~h}}{\rightarrow}} 312 \mathrm{~K} \stackrel{21.4 \mathrm{~h}}{\longrightarrow} 212 \mathrm{~K} \\
& \stackrel{1.4 \mathrm{~h}}{\rightarrow} 301 \mathrm{~K} \underset{\text { Series XVI }}{\stackrel{6.2 \mathrm{~h}}{\rightarrow}} 349 \mathrm{~K} \stackrel{48 \mathrm{~h}}{\longrightarrow} 160 \mathrm{~K} \stackrel{1.8 \mathrm{~h}}{\rightarrow} 310 \mathrm{~K} \underset{\text { Series XVII }}{\stackrel{5 \mathrm{~h}}{\rightarrow}} 349 \mathrm{~K}
\end{aligned}
$$

Other procedural matters are comparable to those described in the previous measurements. $^{(2)}$

\section{Results and discussion}

The experimental heat capacities are given in table 1 and plotted for the transition in figure 1. These results are listed in chronological order. Within a series, the temperature interval of each measurement may usually be inferred from the mean temperatures of adjacent determinations.

The heat capacities were fitted to a series of orthogonal polynomials in regions of normal heat capacity. In the transition region, other methods were used to obtain the total and excess enthalpies and the entropies.

Four presumably first-order transitions were observed in the heat-capacity curve defined by our measurements. The detail of heat-capacity peaks are shown in figure 2. The reproducibility of the heat-capacity curve in our measurements is shown in table 2 for several determinations through this transition region.

Integration of the polynomials and the transitions yielded the thermodynamic 
TABLE 1. Experimental values of heat capacity of thallium(I) $n$-heptanoate $\left(R=8.3144 \mathrm{~J} \cdot \mathrm{K}^{-1} \cdot \mathrm{mol}^{-1}\right)$

\begin{tabular}{|c|c|c|c|c|c|c|c|c|c|}
\hline$T / \mathbf{K}$ & $C_{p, \mathrm{~m}} / \mathrm{R}$ & $T / \mathbf{K}$ & $C_{p, \mathrm{~m}} / R$ & $T / \mathrm{K}$ & $C_{p, \mathrm{~m}} / R$ & $T / \mathrm{K}$ & $C_{p, \mathrm{~m}} / R$ & $T / \mathbf{K}$ & $C_{p, \mathrm{~m}} / R$ \\
\hline \multirow{2}{*}{\multicolumn{2}{|c|}{ Series I }} & 296.50 & 40.31 & 240.85 & 26.50 & 126.84 & 16.61 & 17.56 & 2.113 \\
\hline & & 297.37 & 41.51 & 246.56 & 27.46 & 131.92 & 17.00 & 18.67 & 2.341 \\
\hline 251.26 & 28.38 & 298.22 & 43.62 & 252.12 & 28.65 & 137.02 & 17.36 & 19.78 & 2.572 \\
\hline 257.26 & 29.96 & 299.22 & 44.82 & 255.38 & 28.89 & 142.12 & 17.67 & 20.91 & 2.806 \\
\hline 303.61 & 67.98 & 300.56 & 78.42 & 256.39 & 29.76 & 147.23 & 18.00 & 22.05 & 3.044 \\
\hline 307.85 & 38.59 & 300.92 & 1003 & 257.40 & 29.90 & 152.34 & 18.35 & 23.93 & 3.440 \\
\hline 310.46 & 38.73 & 300.955 & 1620 & 258.40 & 30.59 & 157.45 & 18.70 & 25.87 & 3.859 \\
\hline \multirow{2}{*}{\multicolumn{2}{|c|}{ Series II }} & 300.964 & 1697 & 259.40 & 30.30 & \multirow{2}{*}{\multicolumn{2}{|c|}{ Series XII }} & 27.17 & 4.134 \\
\hline & & 300.997 & 843 & 260.38 & 41.47 & & & 28.52 & 4.415 \\
\hline 286.40 & 34.76 & 301.34 & 69.7 & 261.36 & 36.42 & 155.86 & 18.56 & 29.86 & 4.704 \\
\hline 288.23 & 35.41 & 302.21 & 41.93 & 261.96 & 186.9 & 161.58 & 18.95 & 31.21 & 4.982 \\
\hline 290.69 & 36.31 & 303.27 & 40.85 & 262.11 & 558 & 167.70 & 19.35 & 32,61 & 5.286 \\
\hline$\Delta_{\mathrm{trs}} H_{\mathrm{m}}$ & Detn. A & 304.36 & 39.77 & 262.19 & 480 & 173.84 & 19.74 & 34.07 & 5.563 \\
\hline 305.96 & 38.88 & \multirow{2}{*}{\multicolumn{2}{|c|}{ Series VI }} & 262.28 & 358 & 179.97 & 20.22 & 35.60 & 5.868 \\
\hline 309.05 & 38.56 & & & 262.38 & 267.4 & 186.09 & 20.67 & 37.03 & 6.099 \\
\hline 312.18 & 38.28 & 288.58 & 35.46 & 263.15 & 40.70 & 192.23 & 21.13 & $38.87^{a}$ & 6.664 \\
\hline 315.31 & 38.36 & 290.93 & 36.02 & 264.55 & 30.72 & 198.36 & 21.66 & 41.11 & 6.977 \\
\hline 318.43 & 38.10 & $\Delta_{\mathrm{trs}} H_{\mathrm{m}}$ & Detn. D & 266.08 & 31.30 & 205.37 & 22.24 & 43.35 & 7.350 \\
\hline \multirow{2}{*}{\multicolumn{2}{|c|}{ Series III }} & \multirow{2}{*}{\multicolumn{2}{|c|}{ Series VII }} & 267.44 & 43.57 & 211.51 & 22.86 & 45.57 & 7.789 \\
\hline & & & & 268.30 & 134.7 & 217.63 & 23.48 & 47.95 & 8.181 \\
\hline 284.17 & 34.32 & 295.15 & 41.52 & 268.64 & 307.7 & 223.76 & 24.17 & 50.48 & 8.603 \\
\hline 285.96 & 34.73 & 265.45 & 39.99 & 269.32 & 48.86 & 229.88 & 24.79 & 53.01 & 9.036 \\
\hline 288.34 & 35.17 & 269.98 & 115.9 & 270.25 & 36.15 & 234.22 & 25.53 & \multirow{2}{*}{\multicolumn{2}{|c|}{ Series XV }} \\
\hline 290.68 & 36.21 & $276.85^{a}$ & 15.37 & 270.90 & 40.18 & 236.53 & 25.97 & & \\
\hline$\Delta_{\mathrm{trs}} H_{\mathrm{m}}$ & Detn. B & $283.60^{\circ}$ & 109.2 & 271.27 & 231.2 & $\Lambda_{\mathrm{trs}} H_{\mathrm{m}}$ & Detn. $\mathbf{G}$ & $310.02^{a}$ & 37.84 \\
\hline 305.64 & 38.88 & 285.94 & 34.71 & 271.40 & 285.7 & 276.87 & 33.09 & \multirow{2}{*}{\multicolumn{2}{|c|}{ Series XVI }} \\
\hline 306.90 & 38.72 & 287.68 & 35.41 & 271.83 & 93.06 & 279.86 & 33.60 & & \\
\hline 308.99 & 38.39 & 289.44 & 35.82 & 272.81 & 42.92 & \multirow{2}{*}{\multicolumn{2}{|c|}{ Series XIII }} & 303.11 & 41.04 \\
\hline 312.12 & 38.17 & $\Delta_{\mathrm{trs}} H_{\mathrm{m}}$ & Detn. E & 274.23 & 33.87 & & & 307.81 & 38.62 \\
\hline 315.24 & 38.24 & 305.55 & 38.98 & 275.78 & 33.04 & 50.49 & 8.579 & $313.15^{b}$ & 38.25 \\
\hline 318.36 & 38.19 & $309.50^{a}$ & 44.09 & 277.33 & 33.35 & 53.16 & 9.031 & $318.32^{a}$ & 37.83 \\
\hline \multirow{2}{*}{\multicolumn{2}{|c|}{ Series IV }} & $313.67^{a}$ & 33.36 & \multirow{2}{*}{\multicolumn{2}{|c|}{ Series X }} & 56.08 & 9.474 & $323.47^{a}$ & 38.30 \\
\hline & & \multirow{2}{*}{\multicolumn{2}{|c|}{ Series VIII }} & & & 59.25 & 9.947 & 328.67 & 37.46 \\
\hline 284.19 & 34.39 & & & 233.36 & 25.30 & 62.66 & 10.495 & 333.84 & 37.47 \\
\hline 285.67 & 34.74 & 299,39 & 24.85 & 237.15 & 25.83 & 66.09 & 10.866 & 339.10 & 36.97 \\
\hline 287.42 & 35.18 & 235.36 & 25.66 & 239.20 & 26,00 & 69.77 & 11.378 & 343.82 & 36.52 \\
\hline 289.17 & 36.04 & 241.19 & 26.60 & $\Delta_{\mathrm{trs}} H_{\mathrm{m}}$ & Detn. F & 73.71 & 11.847 & 347.46 & 36.42 \\
\hline 290.92 & 36.43 & 246.89 & 27.55 & 280.18 & 33.61 & 77.66 & 12.312 & \multirow{2}{*}{\multicolumn{2}{|c|}{ Series XVII }} \\
\hline$\Delta_{\mathrm{trs}} H_{\mathrm{m}}$ & Detn. C & 252.45 & 28.70 & \multirow{2}{*}{\multicolumn{2}{|c|}{ Series XI }} & \multirow{2}{*}{\multicolumn{2}{|c|}{ Series XIV }} & & \\
\hline 307.82 & 38.43 & 257.85 & 30.27 & & & & & $314.19^{a}$ & 35.83 \\
\hline 312.87 & 38.19 & 261.32 & 136.13 & 80.97 & 12.72 & $7.54^{a}$ & 0.374 & $320.35^{b}$ & 37.99 \\
\hline \multirow{2}{*}{\multicolumn{2}{|c|}{ Series V }} & 263.79 & 58.39 & 85.01 & 13.17 & 8.73 & 0.482 & $322.95^{a}$ & 15.54 \\
\hline & & 267.02 & 63.30 & 89.00 & 13.60 & 9.83 & 0.649 & $325.70^{a}$ & 39.28 \\
\hline 276.93 & 33.18 & 269.97 & 73.44 & 93.02 & 13.92 & 10.65 & 0.793 & 330.94 & 37.59 \\
\hline 286.15 & 34.76 & 272.80 & 63.19 & 97.06 & 14.23 & 11.51 & 0.948 & 336.27 & 37.24 \\
\hline 289.99 & 35.84 & 276.86 & 33.44 & 101.59 & 14.63 & $12.39^{b}$ & 1.106 & 341.63 & 36.97 \\
\hline 291.36 & 37.13 & 281.81 & 34.02 & 106.63 & 14.98 & 13.30 & 1.267 & $345.66^{a}$ & 36.10 \\
\hline 292.74 & 37.48 & \multirow{2}{*}{\multicolumn{2}{|c|}{ Series IX }} & 111.66 & 15.43 & $14.30^{b}$ & 1.457 & 348.34 & 36.25 \\
\hline 294.08 & 38.15 & & & 116.70 & 15.86 & 15.37 & 1.677 & & \\
\hline 295.40 & 39.40 & 235.01 & 25.63 & 121.76 & 16.27 & 16.45 & 1.889 & & \\
\hline
\end{tabular}

${ }^{a}$ Not used in final fit. ${ }^{b}$ Corrected and used in final fit. 


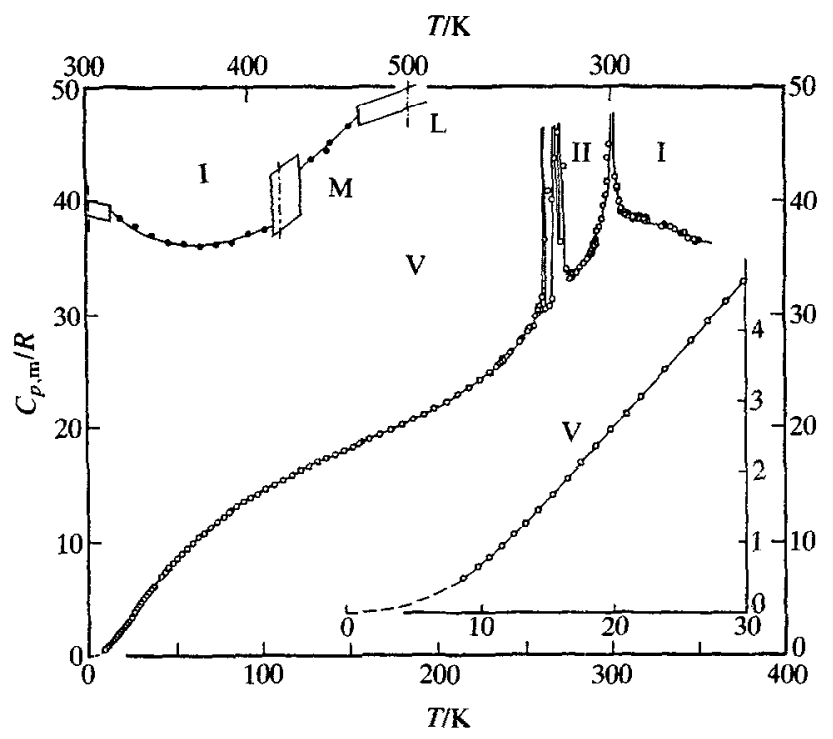

FIGURE 1. Experimental molar heat capacities an constant pressure plotted against temperature for thallium(I) $n$-heptanoate $\mathrm{C}_{6} \mathrm{H}_{13} \mathrm{CO}_{2} \mathrm{Tl}$. $\mathrm{O}$, This work; $\bigcirc$, from reference 2 .

functions. Tabulation of the smoothed values of $C_{p, \mathrm{~m}} / R$ and derived functions is given for selected temperatures in table 3.

In table 4 , a summary of the observed transition temperatures and thermodynamic-property increments and literature values are given for comparison. (The revised numbering of the phases, and designations of the transitions, in table 4 is that adopted in this work.) It can be observed that the sum of the increments of thermal functions of the transitions IV-to-III and III-to-II are almost identical for both adiabatic and d.s.c. techniques, but the individual values of each transition are interchanged.

D.s.c. cooling and heating thermograms $\left(0.17 \mathrm{~K} \cdot \mathrm{s}^{-1}\right)$ given in figure 3 show a thermal hysteresis. The continuous line of the cooling curve consists of three peaks while the corresponding heating curve shows four peaks, instead. The area of the first and the last exothermic peaks correspond to those of the last II-to-I (at $301.9 \mathrm{~K}$ ) and the first V-to-IV (at $262.3 \mathrm{~K}$ ) endothermic peaks, respectively. The second exothermic peak is converted into a bifurcated pair of endothermic peaks (IV-to-III at $267.9 \mathrm{~K}$, and III-to-II at $272.4 \mathrm{~K}$ ) with a small shoulder (at $271.1 \mathrm{~K}$ ), but the areas involved in both cases are approximately the same. On the other hand, another kind of metastability is observed when the cooling step is stopped at about $250 \mathrm{~K}$; once, a second exothermic peak appeared in the cooling thermogram. The heating thermogram is then recorded (dotted curves). Here, the metastable second exothermic peak is converted into two symmetric endothermic peaks (IV'-to-III' at $269.3 \mathrm{~K}$, and III'-to-II at $271.8 \mathrm{~K}$ ), with the total area remaining approximately the same as previously. In addition, the first endothermic peak at $262.3 \mathrm{~K}$ does not 


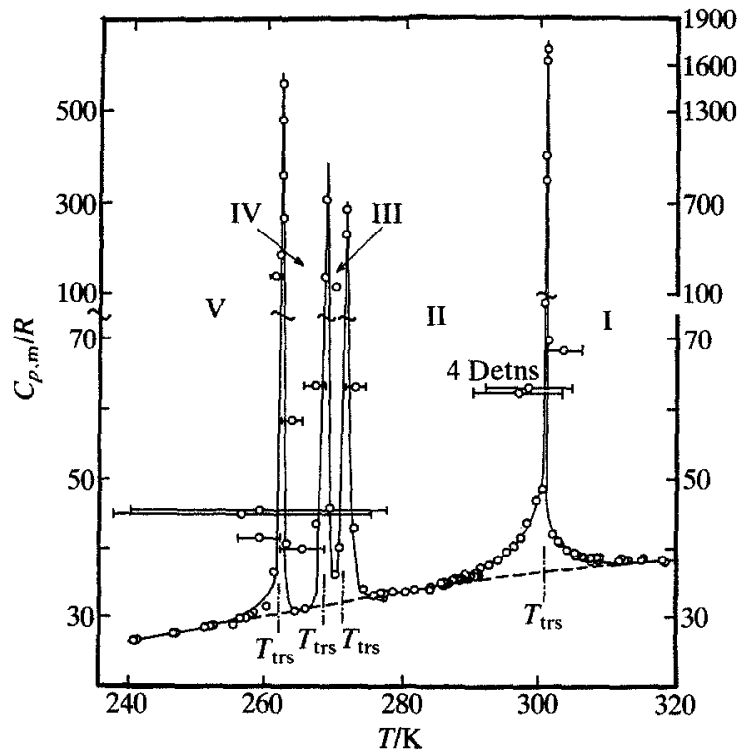

FIGURE 2. Experimental molar heat capacities at constant pressure plotted against temperature through the transition region from 240 to $320 \mathrm{~K}$ for thallium(I) $n$-heptanoate $\mathrm{C}_{6} \mathrm{H}_{13} \mathrm{CO}_{2} \mathrm{Tl}$.

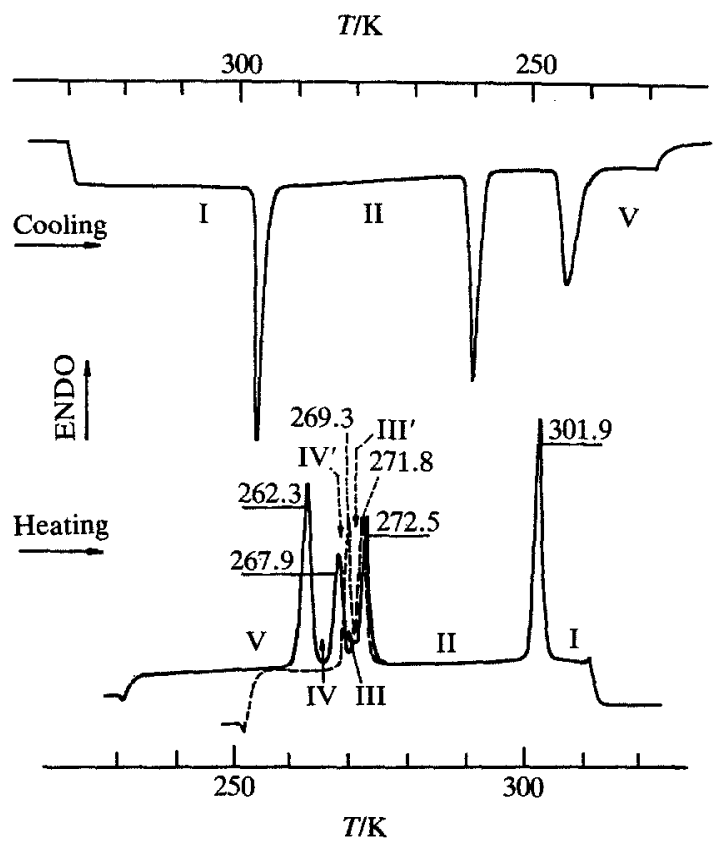

FIGURE 3. D.s.c. cooling and heating thermograms for thallium(I) $n$-heptanoate. 
TABLE 2. Summary of transition thermophysical quantities for thallium(I) $n$-heptanoate $\left(\mathrm{R}=8.3144 \mathrm{~J} \cdot \mathrm{K}^{-1} \cdot \mathrm{mol}^{-1}\right)$

\begin{tabular}{|c|c|c|c|c|c|c|}
\hline Series & $\begin{array}{l}\text { No. of } \\
\text { detns }\end{array}$ & $T_{1} / \mathrm{K}$ & $T_{2} / \mathrm{K}$ & $\Delta_{T_{2}}^{T_{1}} H_{\mathrm{m}}^{\circ} /(R \cdot \mathbf{K})$ & $\Delta_{235 \mathrm{~K}}^{275 \mathrm{~K}} H_{\mathrm{m}}^{\circ} /(R \cdot \mathrm{K})$ & $\Delta_{235 \mathrm{~K}}^{275 \mathrm{~K}} S_{\mathrm{m}}^{\circ} / R$ \\
\hline \multicolumn{7}{|c|}{ Transition sct combining transitions V-to-IV, IV-to-III, and III-to-II } \\
\hline $\begin{array}{l}\text { VIII } \\
\text { X } \\
\text { XII }\end{array}$ & $\begin{array}{c}9 \\
\text { 2+ Detn. F } \\
\text { 1+ Detn. } G\end{array}$ & $\begin{array}{l}238.3 \\
236.12 \\
235.499\end{array}$ & $\begin{array}{l}274.4 \\
277.69 \\
275.37\end{array}$ & $\begin{array}{l}1651.3 \\
1818.0 \\
1756.23\end{array}$ & $\begin{array}{l}1758 \cdot 0 \\
1757_{\cdot 2} \\
1756_{-7}\end{array}$ & \\
\hline \multicolumn{5}{|c|}{ Graphical integration: } & $1757_{-3}^{a}$ & $6.793 \pm 0.003$ \\
\hline \multicolumn{5}{|c|}{ Mean: } & $1757_{-3} \pm 0.7$ & \\
\hline \multicolumn{5}{|c|}{ Lattice contribution: } & $1163.9 \pm 1.0$ & $4.550 \pm 0.004$ \\
\hline \multicolumn{5}{|c|}{$\Delta_{\mathrm{trs}} H_{\mathrm{m}}^{\circ}(\mathrm{V}$-to-IV-to-III-to-II $) /(R \cdot \mathrm{K})=$} & $\overline{593.4 \pm 1}$ & \\
\hline \multicolumn{5}{|c|}{$\begin{aligned} & \Delta_{\mathrm{trs}} S_{\mathrm{In}}^{\circ}(\mathrm{V}-\text { to-IV-to-III-to-II }) / R= \\
& \Delta_{\mathrm{V}}^{\mathrm{V}} H_{\mathrm{m}}^{\circ} /(R \cdot \mathrm{K})=257.2 \pm 1 \Delta_{\mathrm{V}}^{\mathrm{IV}} S_{\mathrm{m}}^{\circ} / R=0.995 \pm 0.004 \\
& \Delta_{\mathrm{IV}}^{\mathrm{II}} H_{\mathrm{m}}^{\circ} /(R \cdot \mathrm{K})=189.7 \pm 1 \Delta_{\mathrm{IV}}^{\mathrm{IIV}} S_{\mathrm{m}}^{\circ} / R=0.707 \pm 0.004 \\
& \Delta_{\mathrm{III}}^{\mathrm{II}} H_{\mathrm{m}}^{\circ} /(R \cdot \mathrm{K})=146.5 \pm 1 \Delta_{\mathrm{III}}^{\mathrm{II}} S_{\mathrm{m}}^{\circ} / R=0.541 \pm 0.003\end{aligned}$} & & $2.243 \pm 0.004$ \\
\hline \multicolumn{5}{|c|}{ Transition II to I } & $\Delta_{290 \mathrm{~K}}^{305 \mathrm{~K}} H_{\mathrm{m}}^{\circ} /(\mathrm{R} \cdot \mathrm{K})$ & $\Delta_{290 \mathrm{~K}}^{305 \mathrm{~K}} S_{\mathrm{m}}^{\circ} / R$ \\
\hline II & $1+$ Detn. A & 289.46 & 304.97 & 909.0 & 890.6 & \\
\hline III & $1+$ Detn. B & 289.51 & 304.89 & 905.1 & 892.8 & \\
\hline IV & $1+$ Detn. $C$ & 290.04 & 304.85 & 884.0 & 891.2 & \\
\hline \multicolumn{5}{|c|}{ Graphical integration: } & $891.2^{a}$ & \\
\hline \multicolumn{5}{|c|}{ Mean: } & $\overline{891.1 \pm 0.6}$ & $2.981 \pm 0.002$ \\
\hline \multicolumn{5}{|c|}{ Lattice contribution: } & $529.2 \pm 1$ & $1.820 \pm 0.004$ \\
\hline \multicolumn{4}{|c|}{$\begin{array}{l}\Delta_{\mathrm{Il}}^{\mathrm{J}} H_{\mathrm{m}}^{\circ} /(R \cdot \mathrm{K})= \\
\Delta_{\mathrm{II}}^{\mathrm{I}} S_{\mathrm{m}}^{\circ} / R=\end{array}$} & & $361.9 \pm 1$ & $1.194 \pm 0.004$ \\
\hline
\end{tabular}

"Not included in the mean value.

appear at all, while the new solid metastable form IV' presents a higher heat capacity than that of the solid stable form V. On tempering the sample at $250 \mathrm{~K}$, the thuscooled sample evolves slowly towards the stable situation.

Compared with the heat-capacity values observed in this study, those of Ngeyi et al..$^{(2)}$ were 2.7 per cent higher below $50 \mathrm{~K}$, about 2.5 per cent higher above $50 \mathrm{~K}$, and more than 5 per cent higher above $300 \mathrm{~K}$. At subambient temperatures, the values of the total enthalpy and entropy for the same set of transitions are about 9 per cent lower than the values observed in this study. These differences, noted in table 5 , are due not only to the absence of acid--and hence of (acid + salt) complex-in the present sample, but also the different evaluation of interpolated lattice heat capacities in transition regions. Taking into account arbitrarily different assignments of lattice heat capacity between the Ngeyi et al. ${ }^{(2)}$ paper and the present work and the inability to resolve exactly the overlapping premonitory and post-transitional effects in the trifucated lower set of transitions, a convincing comparison can be made only by summing the lower set of transitions. When this is done (see table 5) the present measurements are seen to give enthalpy increments about 9 per cent higher, i.e. they are proportional to the amount of salt present. The transition 
TABLE 3. Thermodynamic properties of thallium(I) $n$-heptanoate $\left(R=8.3144 \mathrm{~J} \cdot \mathrm{K}^{1} \cdot \mathrm{mol}^{1}\right)$

\begin{tabular}{|c|c|c|c|c|c|c|c|c|c|}
\hline$\frac{T}{\mathbf{K}}$ & $\frac{C_{p, \mathrm{~m}}}{R}$ & $\frac{\Delta_{0}^{T} S_{\mathrm{m}}^{\circ}}{R}$ & $\frac{\Delta_{0}^{T} H_{\mathrm{m}}^{\circ}}{R \cdot \mathrm{K}}$ & $\frac{\Phi_{\mathrm{m}}^{\circ}(T)}{R}$ & $\frac{T}{\mathrm{~K}}$ & $\frac{C_{p, \mathrm{~m}}}{R}$ & $\frac{\Delta_{0}^{T} S_{\mathrm{m}}^{\circ}}{R}$ & $\frac{\Delta_{0}^{T} H_{\mathrm{m}}^{\circ}}{R \cdot \mathbf{K}}$ & $\frac{\Phi_{\mathrm{m}}^{\circ}(T)}{R}$ \\
\hline \multicolumn{5}{|c|}{ Phase V } & \multicolumn{5}{|c|}{ Phase III } \\
\hline 0 & 0 & 0 & 0 & 0 & $268.64^{a}$ & (308) & $(35.71)$ & $(4827)$ & (17.73) \\
\hline 5 & 0.164 & $(0.055)$ & $(0.205)$ & $(0.014)$ & & {$[31.62]$} & $(34.00]$ & {$[4380]$} & [17.69] \\
\hline 10 & 0.681 & 0.274 & 1.941 & 0.080 & $270^{b}$ & 35.00 & $(35.86)$ & $(4870)$ & $(17.82)$ \\
\hline 15 & 1.600 & 0.720 & 7.594 & 0.214 & & {$[31.88]$} & (34.16] & [4423] & [17.78] \\
\hline 20 & 2.614 & 1.318 & 18.096 & 0.413 & $271.40^{a}$ & $\begin{array}{l}(285) \\
{[32.11]}\end{array}$ & $\begin{array}{l}(36.03) \\
{[34.33]}\end{array}$ & $\begin{array}{l}(4915) \\
{[4468]}\end{array}$ & $\begin{array}{l}(17.92) \\
{[17.87]}\end{array}$ \\
\hline $\begin{array}{l}25 \\
30\end{array}$ & $\begin{array}{l}3.678 \\
4.728\end{array}$ & $\begin{array}{l}2.015 \\
2.779\end{array}$ & $\begin{array}{l}33.818 \\
54.85\end{array}$ & $\begin{array}{l}0.662 \\
0.950\end{array}$ & \multicolumn{5}{|c|}{ Phase II } \\
\hline 40 & 6.733 & 4.416 & 112.24 & 1.610 & $271.40^{a}$ & (285) & (36.57) & $(5061)$ & (17.92) \\
\hline 50 & 8.526 & 6.117 & 188.80 & 2.341 & & {$[32.11]$} & [34.33] & [4468] & [17.87] \\
\hline 60 & 10.069 & 7.811 & 281.93 & 3.112 & 275 & $\begin{array}{l}(32.80) \\
{[32.79]}\end{array}$ & $\begin{array}{l}(36.99) \\
{[34.75]}\end{array}$ & $\begin{array}{l}(5178) \\
{[4585]}\end{array}$ & $\begin{array}{l}(18.16) \\
{[18.08]}\end{array}$ \\
\hline 70 & 11.423 & 9.467 & 389.55 & 3.902 & & & & & \\
\hline 80 & 12.599 & 11.071 & 509.8 & 4.698 & 280 & 33.64 & (37.59) & (5344) & $(18.50)$ \\
\hline 90 & 13.624 & 12.615 & 641.0 & 5.493 & & [33.64] & [35.35] & [4751] & [18.38] \\
\hline 100 & 14.530 & 14.098 & 781.9 & 6.280 & & & & & \\
\hline 120 & 16.10 & 16.89 & 1088.6 & 7.819 & 290 & $\begin{array}{c}35.13 \\
(35.13]\end{array}$ & $\begin{array}{l}(38.80) \\
{[36.56]}\end{array}$ & $\begin{array}{l}(5688) \\
{[5095]}\end{array}$ & $\begin{array}{l}(19.19) \\
{[18.99]}\end{array}$ \\
\hline 140 & 17.51 & 19.48 & 1424.8 & 9.302 & & & & & \\
\hline 160 & 18.86 & 21.91 & 1788.6 & 10.727 & 298.15 & 42.6 & 39.79 & 5979 & 19.74 \\
\hline 180 & 20.24 & 24.21 & 2179.5 & 12.098 & $300.96^{a}$ & $(1700)$ & $(40.13)$ & $(6081)$ & (19.92) \\
\hline 200 & 21.74 & 26.41 & 2598.9 & 13.420 & & {$[36.52\rfloor$} & [37.89] & [5488] & [19.65] \\
\hline 220 & 23.68 & 28.57 & 3052.1 & 14.699 & & & & & \\
\hline & 26.41 & 30.74 & 3551.4 & 15.95 & & & Phase I & & \\
\hline 260 & 29.98 & 32.99 & 4114.2 & 17.17 & $300.96^{a}$ & $(1700)$ & $(41.29)$ & $(6431)$ & (19.92) \\
\hline \multirow[t]{3}{*}{$262.11^{a}$} & (558) & (33.23) & (4178) & $(17.29)$ & & [36.52] & {$[37.89]$} & [5488] & [19.65] \\
\hline & {$[30.41]$} & [33.23] & [4178] & {$[17.29]$} & 310 & $\begin{array}{l}(37.40) \\
\end{array}$ & $\begin{array}{l}(42.38) \\
\end{array}$ & $(6765)$ & $(20.56)$ \\
\hline & & Phase IV & & & 320 & $\begin{array}{c}{[37.40]} \\
38.10\end{array}$ & $\begin{array}{c}{[38.98]} \\
43.59\end{array}$ & $\begin{array}{l}{[3822]} \\
7144\end{array}$ & $\begin{array}{l}{[20.20]} \\
21.26\end{array}$ \\
\hline \multirow[t]{2}{*}{$262.11^{a}$} & (558) & $(34.22)$ & (4435) & (17.29) & & [38.10] & [40.19] & [6201] & [20.81] \\
\hline & {$[30.41]$} & {$[33.23]$} & {$[4178]$} & {$[17.29]$} & 340 & 36.98 & 45.86 & 7896 & 22.64 \\
\hline \multirow[t]{2}{*}{$265^{b}$} & 30.96 & $(34.56)$ & (4523) & (17.49) & 350 & 36.22 & $(46.92)$ & (8261) & (23.32) \\
\hline & [30.96] & [33.57] & [4266] & [17.47] & & & & & \\
\hline \multirow[t]{2}{*}{$268.64^{a}$} & (308) & $(34.99)$ & $(4637)$ & (17.73) & & & & & \\
\hline & [31.62] & {$[34.00]$} & [4380] & [17.69] & & & & & \\
\hline
\end{tabular}

${ }^{a}$ Values in parentheses represent thermodynamic functions for the experimental heat-capacity values on the assumption that they are truly isothermal at $T_{\mathrm{trs}}$. Those in brackets represent the selected lattice (i.e. nontransitional) heat capacity.

${ }^{b}$ Quantities between close or bifurcated transitions when taken as on the lattice curve (see assumption in $a$ ) are also shown in parentheses. Values neither in parentheses or in brackets are thus (real) observed values at the selected temperatures.

previously observed near $296 \mathrm{~K}$ is not present and is presumed to be related to the presence of heptanoic acid on the phase behavior of the binary system - a point requiring further experimentation (which is presently underway). It is clearly not a transition in heptanoic acid as shown by our unpublished work, but is a major transition in the 1-1 (acid + salt) complex (soap) phase system. 
TABLE 4. Summary of transition properties of thallium(I) $n$-heptanoate $\left(R=8.3144 \mathrm{~J} \cdot \mathrm{K}^{-1} \cdot \mathrm{mol}^{-1}\right)$

\begin{tabular}{llcll}
\hline \multicolumn{1}{c}{ Transition } & $T_{\mathrm{trs}} / \mathrm{K}$ & $\Delta_{\mathrm{trs}} H_{\mathrm{m}}^{\circ} /(R \cdot \mathrm{K})$ & $\Delta_{\mathrm{trs}} S_{\mathrm{m}}^{\circ} / R$ & Reference \\
\hline IV'-to-III' & 269.3 & 170 & 0.63 & this work (d.s.c.) \\
III'-to-II & 271.8 & 170 & 0.62 & this work (d.s.c.) \\
V-to-IV & 262.11 & 257.2 & 0.995 & this work (a.c.) \\
& 262.3 & 251 & 0.96 & this work (d.s.c.) \\
IV-to-III & 268.64 & 189.7 & 0.707 & this work (a.c.) \\
& 267.9 & 146 & 0.54 & this work (d.s.c.) \\
III-to-II & 271.4 & 146.5 & 0.541 & this work (a.c.) \\
& 272.4 & 191 & 0.70 & this work (d.s.c.) \\
II-to-I & 300.96 & 361.9 & 1.104 & this work (a.c.) \\
& 301.9 & 319 & 1.06 & 9 \\
I-to-Mesophase & 420.7 & 758 & 1.80 & 9 \\
Mesophase-to-Isotropic & 502.0 & 397 & 0.79 & 9 \\
liquid & & & & \\
\hline
\end{tabular}

TABLE 5. Comparison of cryogenic calorimetric transitions for thallium(I) $n$-heptanoate

\begin{tabular}{lcccc}
\hline $\begin{array}{l}\text { Transition } \\
\text { designation }\end{array}$ & This research & $T_{\text {trs }} / \mathrm{K}$ & \multicolumn{2}{c}{$\Delta_{\mathrm{trs}} H_{\mathrm{m}}^{\circ} /(\boldsymbol{R} \cdot \mathrm{K})$} \\
\hline V-to-IV & Ngeyi et al. ${ }^{(2)}$ & This research $^{a}$ & Ngeyi et al. $^{(2)}$ \\
IV-to-III & $262.1 \pm 0.07$ & $262.8 \pm 0.08$ \\
III-to-II & $268.2 \pm 0.1$ & $267.8 \pm 0.1$ \\
$T_{\text {trs }}^{\text {b }}$ & $271.4 \pm 0.08$ & $271.7 \pm 0.3$ & 593.4 & \\
II-to-I & Not present & $296 \pm 0.8$ & $588^{c}$ & 544 \\
\hline
\end{tabular}

${ }^{a}$ After adjustment of Ngeyi $e t$ al.$^{(2)}$ lattice contribution to correspond to those of the present research.

${ }^{b} T_{\text {trs }}^{y}$ refers to the transition secn by Ngeyi et al ${ }^{(2)}$ near $296 \mathrm{~K}$ and not apparent in the present diagram.

'By d.s.c., this research.

¿ D.s.c. values of 301.9 and $299 \mathrm{~K}$ were determined by Fernández-Martín et al. ${ }^{(9)}$ and by Meisel et al., ${ }^{(11)}$ respectively.

Although the post-transitional heat capacity is significantly different on the II-to-I transition and the estimation of its magnitude suffers from the same difficulties as the lower set, the $\Delta_{\mathrm{trs}} H_{\mathrm{m}}$ seems to be comparable in both sets of measurements.

The apparent differences in peak temperatures of the two sets of transitions is probably an indication of experimental resolution caused by the finite size of the temperature increments of the mapping runs rather than real differences occasioned by purity.

\section{REFERENCES}

1. Boerio-Goates, J.; López de la Fuente, F. L.; Cheda, J. A. R.; Westrutn, E. F., Jr. J. Chem. Thermodynamics 1985, 17, 401.

2. Ngeyi, S. P.; López de la Fuente, F. L.; Cheda, J. A. R.; Fernández-Martín, F.; Westrum, E. F., Jr. J. Chem. Thermodynamics 1985, 17, 409.

3. Ngeyi, S. P.; Westrum, E. F., Jr.; López de la Fuente, F. L.; Cheda, J. A. R.; Fernández-Martín, F. J. Chem. Thermodynamics 1987, 19, 327. 
4. López de la Fuente, F. L.; Cheda, J. A. R.; Westrum, E. F., Jr.; Fernández-Martín, F. J. Chem. Thermodynamics 1987, 19, 1261.

5. Fernández-Martín, F.; López de la Fuente, F. L.; Cheda, J. A. R.; Westrum, E. F., Jr. XXI Bienal Real Sociedad Esp. Quim. (1986).

6. Brouwer, H. W.; Spier, H. L. Thermal Analysis. Proceedings of the Third ICTA at Davos, Switzerland (1971). 1972, Vol. 3, p. 131.

7. McBain, J. W.; Stewart, A. J. Chem. Soc. 1933, 924.

8. McBain, J. W.; Stewart, A. J. Chem. Soc. 1927, 1392.

9. Fernández-Martín, F.; López de la Fuente, F. L.; Cheda, J. A. R. Thermochimica Acta 1983, 73, 109.

10. Franzosini, P.; Sanesi, M. Thermodynamics and Transport Properties of Organic Salts. Pergamon: London. 1980.

11. Meisel, T.; Seybold, K.; Halmos, Z.; Roth, J.; Melykuti, Cs. J. Therm. Anal. 1976, 10, 419.

12. Duruz, J. J.; Michels, M. J.; Ubbelohde, A. R. Proc. Roy. Soc. Lond. A 1971, 322, 781.

13. Sanesi, M.; Franzosini, P. Z. Naturforsch. 1984, 399, 362.

14. Westrum, E. F., Jr.; McCullough, J. P. Experimental Thermodynamics. Scott, D. W.; McCullough, J. P.: editors. Butterworths: New York. 1968, p. 745.

15. Westrum, E. F., Jr. Proceedings NATO Advanced Study Institute on Thermochemistry at Viana do Castello, Portugal. Ribeiro da Silva, M. A. V.: editor. Reidel: New York. 1984, p. 745. 